\title{
Close binary and other variable stars in the solar-age Galactic open cluster M $67^{\star}$
}

\author{
K. Yakut ${ }^{1,2,3}$, W. Zima ${ }^{1}$, B. Kalomeni ${ }^{2,4}$, H. Van Winckel ${ }^{1}$, C. Waelkens ${ }^{1}$, P. De Cat ${ }^{1,5}$, E. Bauwens ${ }^{1}$, M. Vučković ${ }^{1}$, \\ S. Saesen ${ }^{1}$, L. Le Guillou ${ }^{1,6}$, M. Parmaksızoğlu ${ }^{7}$, K. Uluç ${ }^{7}$, I. Khamitov ${ }^{7}$, G. Raskin ${ }^{1}$, and C. Aerts ${ }^{1,8}$ \\ 1 Instituut voor Sterrenkunde, Katholieke Universiteit Leuven, Celestijnenlaan 200D, 3001 Leuven, Belgium \\ 2 University of Cambridge, Institute of Astronomy, Madingley Road, Cambridge CB3 OHA, UK \\ e-mail: yakut@ast.cam.ac.uk \\ 3 University of Ege, Department of Astronomy \& Space Sciences, 35100 Izmir, Turkey \\ 4 Izmir Institute of Technology, Department of Physics, 35430 Izmir, Turkey \\ 5 Royal Observatory of Belgium, Ringlaan 3, 1180 Brussel, Belgium \\ ${ }^{6}$ LPNHE - Université Paris, 75252 Paris Cedex 05, France \\ 7 TÜBİTAK National Observatory, Antalya, Turkey \\ 8 IMAPP, Radboud Universiteit Nijmegen, PO Box 9010, 6500 GL Nijmegen, The Netherlands
}

Received 21 February 2009 / Accepted 5 June 2009

\section{ABSTRACT}

\begin{abstract}
We present multi-colour time-series CCD photometry of the solar-age galactic open cluster M67 (NGC 2682). About 3600 frames spread over 28 nights were obtained with the $1.5 \mathrm{~m}$ Russian-Turkish and $1.2 \mathrm{~m}$ Mercator telescopes. High-precision observations of the close binary stars AH Cnc, EV Cnc, ES Cnc, the $\delta$ Scuti type systems EX Cnc and EW Cnc, and some long-period variables belonging to M 67 are presented. Three full multi-colour light curves of the overcontact binary AH Cnc were obtained during three observing seasons. Likewise we gathered three light curves of EV Cnc, an EB-type binary, and two light curves of ES Cnc, a blue straggler binary. Parts of the light change of long-term variables S1024, S1040, S1045, S1063, S1242, and S1264 are obtained. Period variation analysis of $\mathrm{AH} \mathrm{Cnc,} \mathrm{EV} \mathrm{Cnc,} \mathrm{and} \mathrm{ES} \mathrm{Cnc} \mathrm{were} \mathrm{done} \mathrm{using} \mathrm{all} \mathrm{times} \mathrm{of} \mathrm{mid-eclipse} \mathrm{available} \mathrm{in} \mathrm{the} \mathrm{literature} \mathrm{and} \mathrm{those}$ obtained in this study. In addition, we analyzed multi-colour light curves of the close binaries and also determined new frequencies for the $\delta$ Scuti systems. The physical parameters of the close binary stars were determined with simultaneous solutions of multicolour light and radial velocity curves. Finally we determined the distance of M 67 as 857(33) pc via binary star parameters, which is consistent with an independent method from earlier studies.
\end{abstract}

Key words. binaries: close - binaries: eclipsing - stars: oscillations - open clusters and associations: individual: M 67

\section{Introduction}

The open cluster M 67 (NGC 2682) is located at a distance of $\sim 840 \mathrm{pc}$ and its stellar content consists of stars of solar age and composition. Van den Berg \& Stetson (2004) give an isochrone age of 4.0 Gyr using the working hypothesis that isochrones that treat overshooting are able to reproduce the morphology of the cluster turnoff. Hurley et al. (2005) present a direct $N$-body model of M 67 from zero to $4 \mathrm{Gyr}$ age. The authors not only take the cluster dynamics into account but also the evolution of stars and binary systems. The $N$-body model's initial setup for a given cluster is a statistical realisation where the mass, the position, and the velocity of a star are deduced from a distribution function. The model of Hurley et al. (2005) contains 20 blue stragglers at 4 Gyr, nine of which are in binaries. Tian et al. (2006) present simulations for the primordial blue stragglers belonging to M67. Four of them had been predicted by their simulations using appropriate parameters.

Besides evolved red giants, the HR diagram of M 67 contains main-sequence stars. The mass of the stars that reside near

\footnotetext{
* Based on observations made with the Mercator Telescope, operated on the island of La Palma by the Flemish Community, at the Spanish Observatorio del Roque de los Muchachos of the Instituto de Astrofísica de Canarias and TÜBITAK National Observatory Russian-Turkish telescope operated at Saklıkent, Antalya.
}

the turnoff point provides a crucial astrophysical tool for testing the predictions of stellar evolution models. Several clusters show that a group of hot stars known as blue stragglers still appear to be on the main sequence above the turnoff point. These stars are therefore hotter and bluer than other cluster member stars with the same luminosity. They may be regarded as unique astrophysical objects in the study of stellar evolution theories since they show an evolution stage unexpected by standard theories. The current scenarios for explaining the blue stragglers are: (1) post-mass transfer binaries; (2) merged former binaries; or (3) the products of dynamical collisions. All of these models produce rejuvenated main sequence stars. The merging of two stars would create a hot, massive, and more luminous star with the same age; hence, blue straggler stars would have more hydrogen in their cores, making them appear much younger star than they are. This hypothesis can in principle be tested by studying the pulsation properties of blue straggler stars whose asteroseismological properties are expected to be different from those of normal pulsating stars with similar masses and luminosities.

The solar age cluster M 67 is expected to contain active stars similar to our Sun, and some binaries belonging to the cluster (e.g. ES Cnc) are found to be chromospherically active stars based on the observed $\mathrm{Ca}$ II H-K lines in their spectrum. For instance, AG Cnc (S113) (Kaluzny \& Radczynska 1991) was 
found to be a low-temperature contact (WUMa-type) binary. Recently, Giampapa et al. (2006) studied the chromospheric activity of solar-like stars in M67. The authors studied Ca II H and $\mathrm{K}$ lines of 60 stars. Giampapa et al. (2006) predict the age of the open cluster M67 to be on the range 3.8-4.3 Gyr. In the cluster M67, FK Com type giant stars and white dwarfs are also found. Landsman et al. (1998) reported that the number of white dwarfs (WD) in M67 is consistent with those expected from WD cooling models. X-ray observations of the cluster reveal somewhat special stars of the cluster. ROSAT X-ray observations of M 67 indicate that active synchronized binaries have rapid rotation despite their rather advanced ages. A magnetic cataclysmic variable star EU Cnc, due its lower visual magnitude, is identified with ROSAT observations. The photometric variable stars S1063 and S113 belonging to M 67, independently identified as X-ray sources by ROSAT (Belloni et al. 1993; Belloni et al. 1998), are also reported to be a spectroscopic binary by Mathieu et al. (2003).

$\mathrm{AH} \mathrm{Cnc}\left(V_{\mathrm{mag}}=13.33, \mathrm{~F} 7 \mathrm{~V}\right)$ is a low-temperature contact binary (LTCB) system. The variability of AH Cnc has been reported by Kurochkin (1960) who classified it as an RR Lyrae type variable. Later on, the system was classified as WUMa-type by Efremov et al. (1964) and Kurochkin (1965). Though the system has been studied photometrically by numerous researchers, the radial velocity curve of the system was obtained only by Whelan et al. (1979). The mass ratio obtained from a spectroscopic study of the system displays a difference from those obtained by photometric studies. Previous modelling of the system led to orbital and physical parameters and was based on single colour RTT150 data (Yakut \& Aerts 2006).

EV Cnc was discovered to be a contact binary by Gilliland et al. (1991). The reported period of the system is 0.44125 days (ibid). van den Berg et al. (2002) published the light curve of the contact binary through partial eclipses and ellipsoidal variations due to tidal effects of the components. The observed X-ray emission from the system is believed to be due to the coronal activity of the magnetically active component. The observed small amplitude light variation is due to the small inclination of the system or to the high mass ratio of the component stars (van den Berg et al. 2002). WUMa-type light curve properties related with unequal eclipses, were visible in the light curve of van den Berg et al. (2002), i.e., at phase 0.25 the system is brighter than it is at phase 0.75 . This feature has been explained with a hot spot activity on the secondary star. Blake \& Rucinski (2004) tested a technique of combining Fourier coefficients of the light curves with spectroscopically obtained mass ratios to provide distance calculations to the binary. However, EV Cnc was found to be unsuitable for such distance determination due to its faintness.

ES Cnc (S1082) is classified as a member of the RS CVn type binaries, which are detached and active systems. Their evolution is mainly driven by angular momentum loss by a stellar wind. ES Cnc (S1082), with its orbital period of 1.08 days, falls in the blue part of the colour-magnitude (CM) diagram. ES Cnc is an unusual partial-eclipsing blue straggler star. Its light curve obtained by Sandquist \& Shetrone (2001) was reported to show variations on timescales of a month and shorter. Sandquist et al. (2003) showed the variation of the system in the $V$ band to be in the range $0.01-0.03 \mathrm{mag}$ from month to month. Such variations are also detected in our new observations of the binary. Sandquist et al. (2003) explained them as due to spots on the cold and faint component star. The authors also showed the existence of a third body orbiting the binary in a highly eccentric $(e=$ $0.6)$ orbit with a 1189 days period. The primary and the third components are blue stragglers (Sandquist \& Shetrone 2001). The stars were identified from their systematic velocities as members of M 67. The spectroscopic detection of a binary companion to the blue straggler S1082 was confirmed from highresolution spectra. The measured projected rotational speed of the component is $90 \pm 20 \mathrm{~km} \mathrm{~s}^{-1}$ and its radial velocity was found to vary with a peak-to-peak amplitude of $\leq 25 \mathrm{~km} \mathrm{~s}^{-1}$. The radial velocity period of the system does not correspond with the period derived from partial eclipses. Therefore, it is believed that the system undergoes mass transfer (Shetrone \& Sandquist 2000). The $V$-band light curve of the system shows brightness variations due to spot activity of the secondary cool component which is nearly synchronized (Sandquist et al. 2003).

Gilliland et al. (1991) studied for the first time the $\delta$ Scuti type pulsating properties of EW Cnc and EX Cnc, members of M 67. Gilliland \& Brown (1992) detected 10 independent modes for EW Cnc and 5 modes for EX Cnc. Recently, these stars were studied by Sandquist \& Shetrone (2003) and Zhang et al. (2005). Zhang et al. (2005) detected 4 modes for EW Cnc and 5 modes for EX Cnc. A spectroscopic study was performed by Milone \& Latham (1992) and they showed that the system is indeed a binary with a period of 4.2 days and an eccentricity $e=0.2$.

\section{New observations, data reduction, and new light curves}

CCD observations of M67 were obtained with the $1.5 \mathrm{~m}$ Russian-Turkish telescope (RTT150) at TÜBITAK National Observatory (TUG, Antalya, Turkey) and with the $1.2 \mathrm{~m}$ Mercator telescope at Roque de los Muchachos Observatory on La Palma Island (Canary Islands, Spain). The cluster has been observed on 5 nights in 2005 with the $1.5 \mathrm{~m}$ telescope and on 23 nights in 2006 with the $1.2 \mathrm{~m}$ telescope. The journal of the observations is given in Table 1.

TUG observations were obtained with the ANDOR CCD on the RTT150. The CCD chip has $2 \mathrm{~K} \times 2 \mathrm{~K}$ pixels and the corresponding field of view is $8.2^{\prime} \times 8.2^{\prime}$. The gain corresponds to $1.2 \mathrm{e}^{-} / \mathrm{ADU}$ with readout noise of 2 electrons rms. During the observations a Cousins $R_{c}(670 \mathrm{~nm})$ filter was used. The $2 \mathrm{~K} \times 2 \mathrm{~K}$ MEROPE CCD camera was used during observations with the Mercator telescope. The gain corresponds to $0.93 \mathrm{e}^{-} / \mathrm{ADU}$ with a readout noise of 4.5 electrons rms and the corresponding field of view is $6.5^{\prime} \times 6.5^{\prime}$. The $V(\mathrm{VG}-548.26 \mathrm{~nm}), R(\mathrm{RG}-662.91 \mathrm{~nm})$, and $I$ (IC-749.18 nm) filters were used. The exposure times were $15 \mathrm{~s}$ for $V, 7.5 \mathrm{~s}$ for $R, 10 \mathrm{~s}$ for $I$ and, $2 \mathrm{~s}$ for $R_{\mathrm{C}}$ filter.

During the data reduction, we have studied all the nights and each frame separately. The reduction and analysis of the frames have been performed using the IRAF packages to subtract the standard bias, dark, and divide flat-field, followed by aperture photometry. 10 comparison stars that were assured in the previous studies were used. These comparison stars are GSC $8141205\left(C_{1}\right)$, GSC $8141425\left(C_{2}\right)$, GSC $8141311\left(C_{3}\right)$, GSC $8141146\left(\mathrm{C}_{4}\right)$, GSC $8141981\left(\mathrm{C}_{5}\right)$, GSC $8141631\left(\mathrm{C}_{6}\right)$, GSC $8141205\left(\mathrm{C}_{7}\right)$, GSC $8141205\left(\mathrm{C}_{8}\right)$, GSC $8141205\left(\mathrm{C}_{9}\right)$, and GSC $8141205\left(\mathrm{C}_{10}\right)$. For the Mercator observations only six of them were used.

We observed the cluster on 28 nights, most of which successive. The data from the successive nights revealed short timescale light variations of AH Cnc, EV Cnc, and ES Cnc. Variable stars falling inside the CCD frame are shown on the CM diagram in Fig. 1. Some parameters of the variables are listed in Table 2. $V, B-V$, and orbital periods given in the table are obtained from Montgomery et al. (1993) and van den Berg et al. (2002). 
Table 1. Summary of the observations of M 67.

\begin{tabular}{lllllc}
\hline \hline Run & UT date & JD $^{*}$ & Telescope & Filter & $N_{\text {Obs }}$ \\
\hline 1 & 2005 February 23 & $425.36-425.50$ & RTT150 & $R_{\mathrm{C}}$ & 270 \\
2 & 2005 February 24 & $426.26-426.58$ & RTT150 & $R_{\mathrm{C}}$ & 464 \\
3 & 2005 April 28 & $489.26-429.40$ & RTT150 & $R_{\mathrm{c}}$ & 205 \\
4 & 2005 November 7 & $482.36-482.66$ & RTT150 & $R_{\mathrm{C}}$ & 166 \\
5 & 2005 November 8 & $683.50-683.66$ & RTT150 & $R_{\mathrm{c}}$ & 284 \\
6 & 2006 January 7 & $743.57-743.71$ & Mercator & $V R I$ & 288 \\
7 & 2006 January14 & $750.56-750.80$ & Mercator & $V R I$ & 504 \\
8 & 2006 January 19 & $755.42-755.49$ & Mercator & $V R I$ & 102 \\
9 & 2006 April 29 & $855.39-855.47$ & Mercator & $V R I$ & 18 \\
10 & 2006 May 3 & $859.40-859.46$ & Mercator & $V R I$ & 123 \\
11 & 2006 May 4 & $860.39-860.47$ & Mercator & $V R I$ & 143 \\
12 & 2006 May 5 & $861.39-861.47$ & Mercator & $V R I$ & 147 \\
13 & 2006 May 6 & $862.40-862.51$ & Mercator & $V R I$ & 123 \\
14 & 2006 May 7 & $863.39-863.47$ & Mercator & $V R I$ & 147 \\
15 & 2006 May 8 & $864.41-864.47$ & Mercator & $V R I$ & 69 \\
16 & 2006 May 9 & $865.40-865.47$ & Mercator & $V R I$ & 69 \\
17 & 2006 May 10 & $866.39-866.46$ & Mercator & $V R I$ & 72 \\
18 & 2006 May 11 & $867.40-867.46$ & Mercator & $V R I$ & 63 \\
19 & 2006 May 12 & $868.39-868.45$ & Mercator & $V R I$ & 66 \\
20 & 2006 May 13 & $869.41-869.45$ & Mercator & $V R I$ & 54 \\
21 & 2006 May 14 & $870.41-870.46$ & Mercator & $V R I$ & 18 \\
22 & 2006 May 15 & $871.43-871.44$ & Mercator & $V R I$ & 6 \\
23 & 2006 May 16 & $872.40-872.44$ & Mercator & $V R I$ & 45 \\
24 & 2006 May 17 & $873.40-873.43$ & Mercator & $V R I$ & 27 \\
25 & 2006 May 19 & $875.40-874.44$ & Mercator & $V R I$ & 33 \\
26 & 2006 May 20 & $876.41-876.43$ & Mercator & $V R I$ & 24 \\
27 & 2006 May 21 & $877.40-877.43$ & Mercator & $V R I$ & 30 \\
28 & 2006 May 23 & $879.40-879.41$ & Mercator & $V R I$ & 36 \\
\hline & & & & &
\end{tabular}

JD* lists JD start+2 453000 .

Figure 1 shows that $\mathrm{AH} \mathrm{Cnc}$ and EV Cnc are at the turn-off point of the cluster, whereas ES Cnc, EW Cnc, and EX Cnc lay in the blue part of the diagram. The light curves of AH Cnc obtained with both telescopes are shown in Fig. 2a-c. The light curve of AH Cnc obtained by Whelan et al. (1979), using different telescopes and photoelectric systems, does not show flat secondary minima as the light curve obtained in this study. The difference in the light curves remains unexplained. The multi-colour observations of the total eclipse light curves allow us to determine accurate orbital parameters of the binary and the physical parameters of the components. The orbital phases are calculated by using Eq. (3).

The light variations of EV Cnc obtained with the Mercator telescope and RTT150 are shown in Fig. 3. The phases are calculated using Eq. (4). The light curve obtained in May 2006 shows the full light variation of the binary. Although the phase coverage of the Mercator observations made in January and that of the RTT150 observations is not complete, these light curves are still useful. The observed difference in the maxima levels are in agreement with the observations given in the literature (see, van den Berg et al. 2002). We observe asymmetry during the ingress and egress of the secondary minimum. We will discuss it again in the last section. The difference in minima is conspicuous in the light curve of EV Cnc since the system is of $\beta$ Lyr type rather than WUMa type. The light variations of ES Cnc, obtained with the Mercator telescope are shown in Fig. 4. The phases are calculated using the new ephemeris given by Eq. (6). The observations of the system made in May (Fig. 4) cover the full phase, so it gives the opportunity to study its light curve in detail. The levels of max I and max II shown in Fig. 4 are different. This is believed to be an activity indicator of the system.

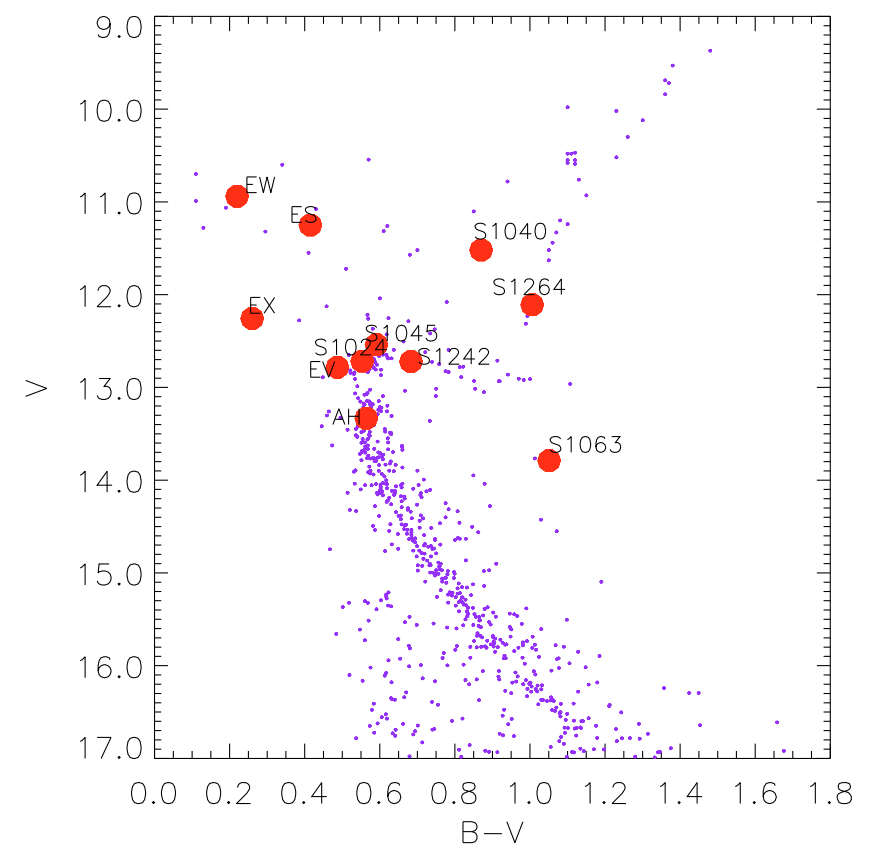

Fig. 1. The colour magnitude diagram of open cluster M 67. The colours and the magnitudes of the stars are obtained from Montgomery et al. (1993).

Long-period variables (S1024, S1040, S1045, S1063, $\mathrm{S} 1242$, and S1264) that fall inside the CCD frame are given in Table 2. The shortest period system is S1024 (7.16 days) and the longest one with 354 days is S1264. In the CM diagram of the open cluster, the systems S1063 and S1113 lay well below the subgiant branch. Mathieu et al. (2003) studied these subgiant members of M 67 spectroscopically and obtained their orbital parameters. S1063 is a single-lined binary with an orbital period of 18.4 days and an eccentricity 0.21 . The system $\mathrm{S} 1113$ is a double-lined binary and it has a circular orbit with 2.8 days period. During the observations made with the Mercator telescope the systems did not fall inside the CCD and with the RTT150 the system S1063 fell within the CCD frame. Their long periods require long term observations of them to retrieve information from their light curves. The light variation of S1040 obtained in May, 2006 with the Mercator telescope is shown in Fig. 5a. The light variations of S1024, S1045, and S1264 are shown in Fig. $5 \mathrm{~b}-\mathrm{d}$. The observed variations in these systems are not conspicuous as in the case of S1040 (Fig. 5a) though small amplitude variations are still detectable. We do not have enough data to analyze these long period variables, therefore, new observations of them are important for evolutionary study of them.

\section{Data analysis}

\subsection{New ephemeris and period change analysis of close binaries}

The orbital period of a binary system can vary because of mass transfer, mass loss, the presence of a third body etc. If the variations are large enough they can be studied in O-C $\left(\Delta T=T_{\mathrm{o}}-T_{\mathrm{c}}\right)$ diagrams. The mass transfer between the components show a parabolic variation in the $\mathrm{O}-\mathrm{C}$ diagram, a third body in the system represent a sinusoidal variation. Hence, a sinusoidal variation superimposed on an upward/downward parabola suggests mass transfer between the components of a binary system that 
Table 2. Variable stars in M 67 that fall inside CCD frames.

\begin{tabular}{llllllllll}
\hline \hline Star name & S no. & MMJ no. & Type & Sp.T. & $\alpha_{2000}$ & $\delta_{2000}$ & $m_{V}$ & $B-V$ & Period (days) \\
\hline AH Cnc & 1282 & 6027 & C & F6.5V & 085138 & +115057 & 13.33 & 0.56 & 0.360 \\
EV Cnc & 1036 & 5833 & SD & F3V & 085128 & +114928 & 12.78 & 0.49 & 0.441 \\
ES Cnc & 1082 & 6493 & RS CVn & F4V & 085121 & +115326 & 11.25 & 0.42 & 1.068 \\
EX Cnc & 1284 & 6504 & $\delta$ Sct & A7V & 085134 & +115111 & 10.94 & 0.22 & \\
EW Cnc & 1280 & 5940 & $\delta$ Sct & & 085133 & +115041 & 12.26 & 0.26 & \\
& 1024 & 5739 & & & 085135 & +115032 & 12.72 & 0.55 & 7.160 \\
& 1040 & 6488 & & G4III & 085135 & +115032 & 11.52 & 0.87 & 42.88 \\
& 1045 & 5654 & & & 085135 & +115032 & 12.54 & 0.59 & 7.645 \\
& 1063 & 5542 & & G8IV & 085135 & +115032 & 13.79 & 1.05 & 18.39 \\
& 1242 & 5993 & & & 085135 & +115032 & 12.72 & 0.68 & 31.78 \\
& 1264 & 5877 & & & 085135 & +115032 & 12.09 & 0.94 & 353.9 \\
\hline
\end{tabular}
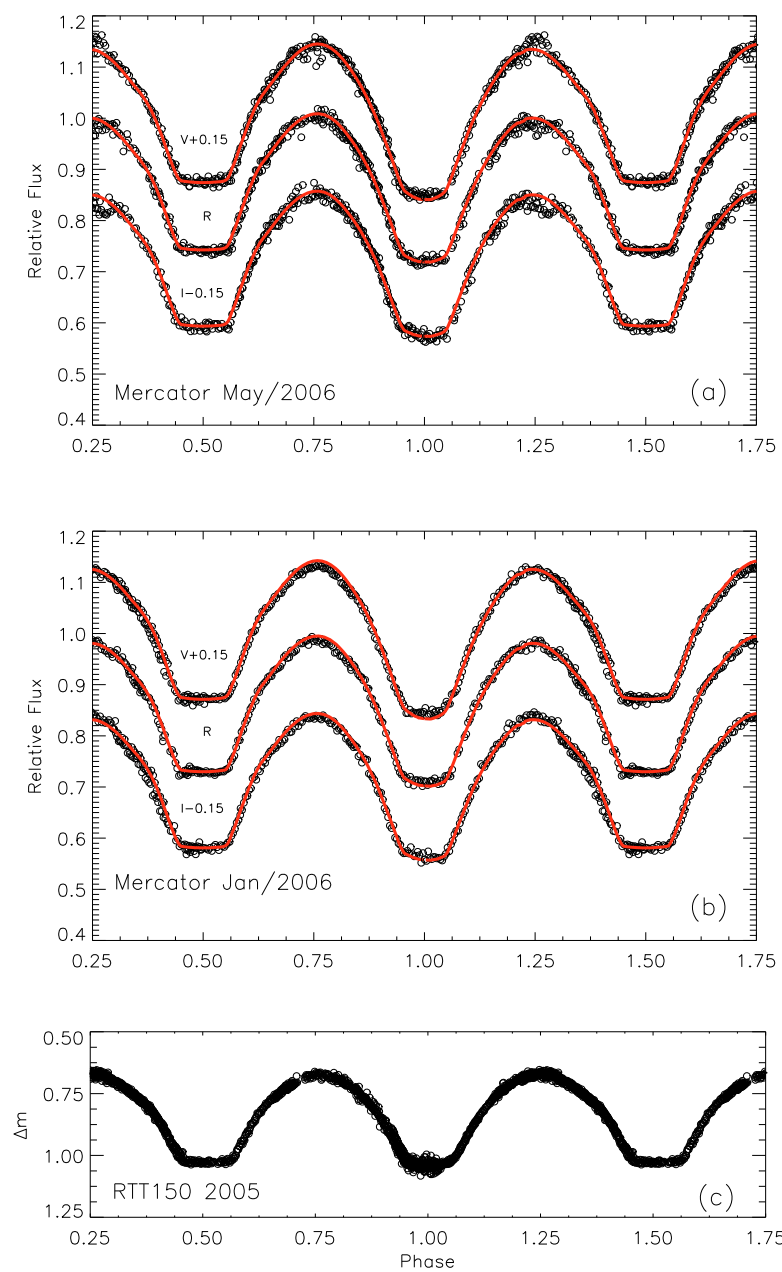

Fig. 2. The observed and computed (solid line) light curves of AH Cnc. For the sake of comparison the light curves in $V$ and $I$ bands are shifted by a value of $+0.15,-0.15$ in relative flux.

has a third body orbiting it e.g. XY Leo (Yakut et al. 2003), TY Boo (Yang et al. 2007), V839 Oph (Akalin \& Derman 1997), BB Peg (Kalomeni et al. 2007). Usually, ephemeris of such a system is written as

$\operatorname{Min} \mathrm{I}=T_{\mathrm{o}}+P_{\mathrm{o}} E+\frac{1}{2} \frac{\mathrm{d} P}{\mathrm{~d} E} E^{2}+\tau$,
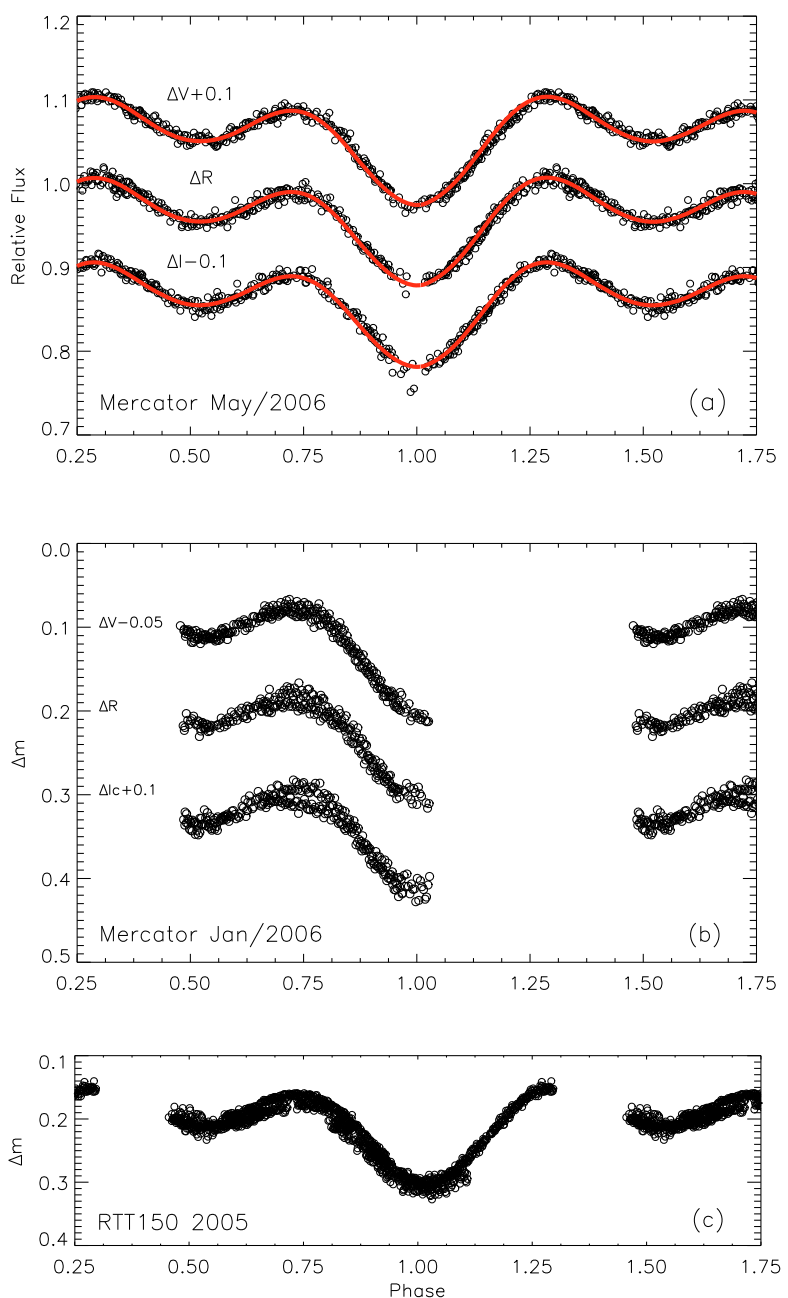

Fig. 3. Same as Fig 2, but for EV Cnc. $V$ and $I$ bands are shifted by a value of +0.1 and -0.1 in relative flux.

where the first two terms are linear, the third one is due to the period increase/decrease and the last term $\tau$, is the light-time term, which was formalized by Irwin (1952) as

$\tau=\frac{a_{12} \sin i^{\prime}}{c}\left[\frac{1-\mathrm{e}^{\prime 2}}{1+\mathrm{e}^{\prime} \cos v^{\prime}} \sin \left(v^{\prime}+\omega^{\prime}\right)+\mathrm{e}^{\prime} \sin \omega^{\prime}\right]$.

In Eq. (1), $T_{\mathrm{o}}$ is the starting epoch for the primary minimum, $E$ is the integer eclipse cycle number, $P_{\mathrm{O}}$ is the orbital period of the eclipsing binary. In Eq. (2), $a_{12}, i^{\prime}, e^{\prime}$, and $\omega^{\prime}$ are the semi-major 


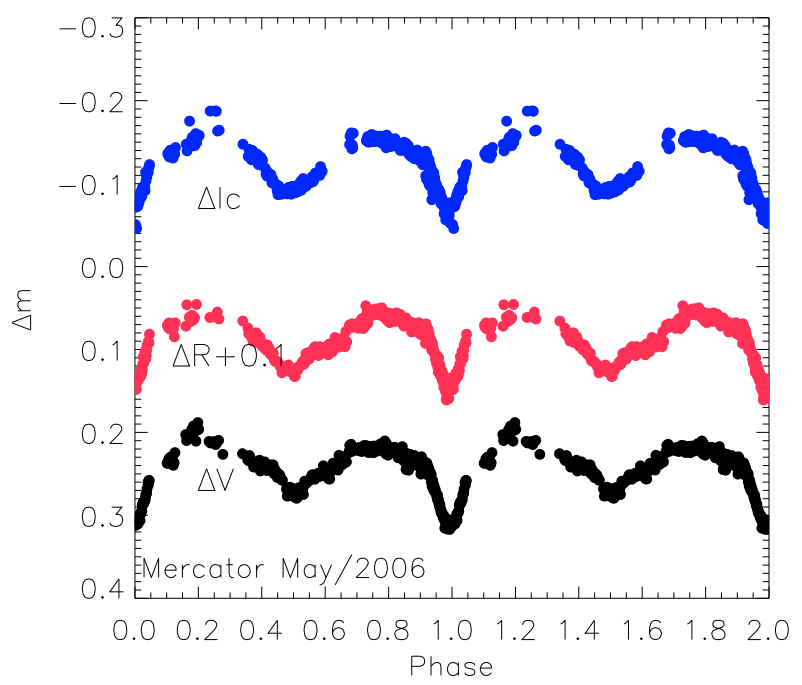

Fig. 4. Same as Fig. 2 but for RS CVn type binary ES Cnc.

axis, inclination, eccentricity, and the longitude of the periastron of the eclipsing pair around the third body, and $v^{\prime}$ is the true anomaly of the position of the center of mass, time of periastron passage $T^{\prime}$ and orbital period $P^{\prime}$ are the unknown parameters in Eq. (2).

\subsubsection{The low-temperature contact binary AH Cnc (S1282)}

In this study, we obtained six minima times for AH Cnc. They are listed in Table 3 with their errors. All the available times of light minimum of $\mathrm{AH} \mathrm{Cnc}$ were collected from the literature and are listed in Table 4. We improved the new linear ephemeris as:

Min I = HJD $2453750 \mathrm{~d} \cdot 6239(7)+0.3604583(2) \times E$.

In an earlier study, Qian et al. (2006) presented 50 minima times, while we collected 76 times of minima. We merged all these data to analyze the $\mathrm{O}-\mathrm{C}$ variation. For the sake of comparison with Qian et al. analysis, differences between the observed and calculated times $(\Delta T)$ were estimated using the ephemeris given by Kriener et al. (2001). They are plotted versus time in Fig. 6. The $(\Delta T)_{I}$ values show a parabola-like curve (Fig. 6) which indicates the existence of mass transfer in the system. The upward parabola shows that the mass transfer occurs from the less massive component to the more massive one.

Recently, the existence of a third body was reported by Qian et al. (2006) and Pribulla \& Rucinski (2006). We used the linear ephemeris to construct the binary's O-C diagram. It shows almost a sine-like variation superposed on an upward parabola. A sine-like variation in the O-C curve, where both primary and secondary minima follow the same trend, suggests a light time effect due to the presence of a tertiary component.

The light elements from Eq. (1) and Eq. (2) were determined using the differential correction method. We used these equations with the values given in Table 4 and a weighted least squares solution to derive the parameters shown in Table 5. The $1 \sigma$ standard errors, in the last digit are given in parentheses. We assigned a weight of 5 to the photoelectric (pe) and CCD observations, 1 to the photographic (pg) measurements. The $\left(\Delta T_{\mathrm{I}}\right)$ variation of the system is plotted in Fig. 6a. The dashed line in Fig. 6a shows the secular increase of the binary's orbital period while the solid line is both the secular increase and the
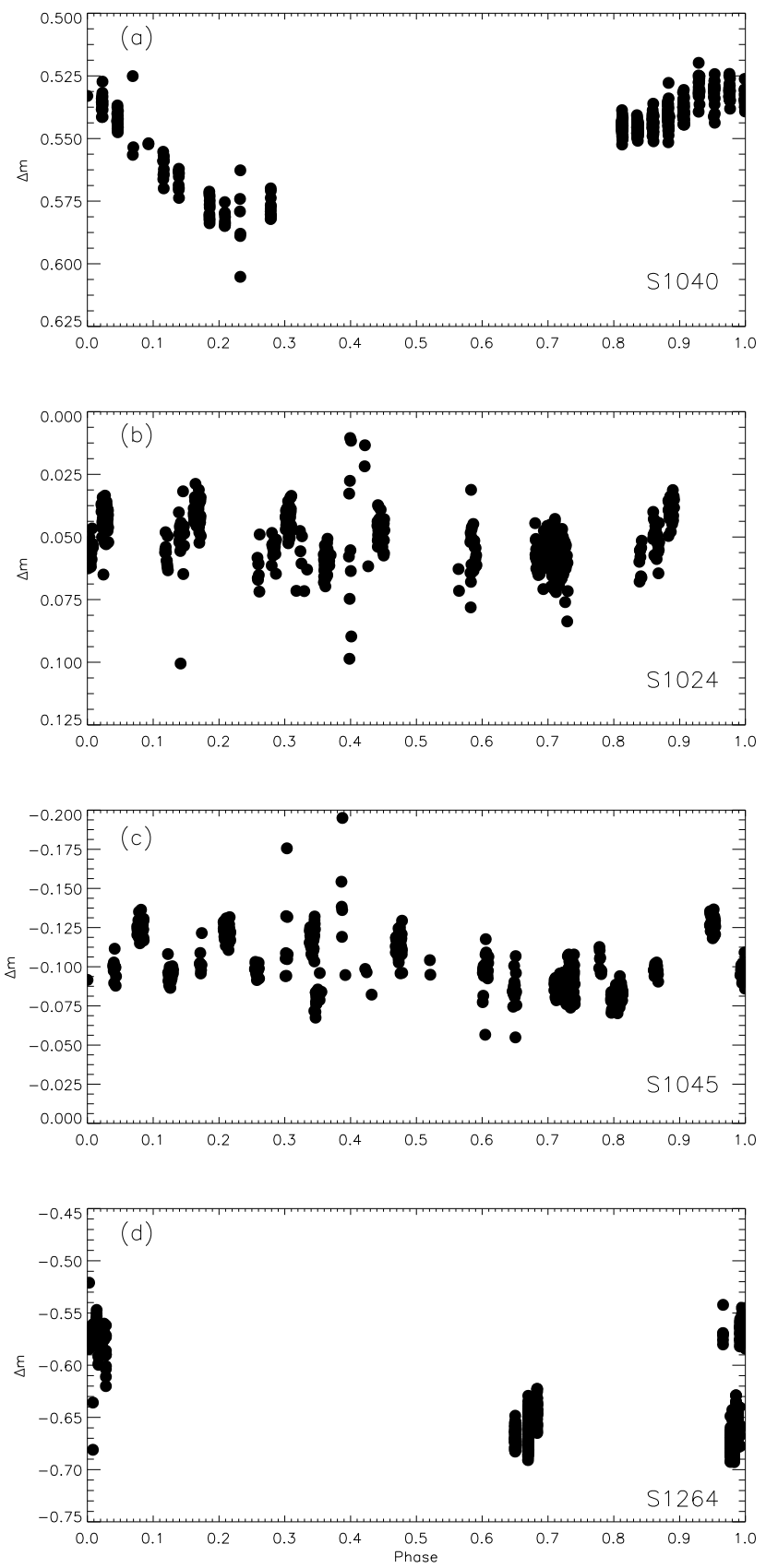

Fig. 5. Observed $V$ light curves of a) S1040; b) S1024; c) S1045; and d) $\mathrm{S} 1264$.

light-time effect of the tertiary component. A period increase $\mathrm{d} P / \mathrm{d} t=+3.69(6) \times 10^{-7} \mathrm{~d} \mathrm{yr}^{-1}$ is found. The contribution of the light-time effect, $(\Delta T)_{\mathrm{II}}$, to the total period variations of the system is shown in Fig. $6 b$.

\subsubsection{The near-contact binary EV Cnc (S1036)}

No period variation analysis of EV Cnc has been presented in literature. We collected all available times of minimum light from the literature (see Table 6) and added our new times of minima (Table 3). The linear ephemeris of the system were improved as:

Min I = HJD 2453 682.6039(7) $+0.441437(6) \times E$. 
Table 3. New times of the primary and secondary minima for AH Cnc and EV Cnc in HJD-2 400000.

\begin{tabular}{llll}
\hline \hline & Times of minima & Error & Filter \\
\hline AH Cnc & & & \\
& 53426.39314 & \pm 0.00008 & $R_{c}$ \\
& 53489.29283 & \pm 0.00008 & $R_{c}$ \\
& 53683.58142 & \pm 0.00006 & $R_{c}$ \\
& 53750.62330 & \pm 0.00025 & $I$ \\
& 53750.62366 & \pm 0.00019 & $V$ \\
& 53750.62366 & \pm 0.00014 & $R$ \\
EV Cnc & & & \\
& 53425.4532 & \pm 0.0003 & $R_{c}$ \\
& 53426.3392 & \pm 0.0002 & $R_{c}$ \\
\hline
\end{tabular}

Since the system is a semi detached (or contact), continuing mass-transfer between the components should affect the O-C variation. If the mass transfer is large enough, a parabolic variation in $\mathrm{O}-\mathrm{C}$ diagram can be detected. Figure 7 indicates such a period increase over time. To obtain the light elements in Eq. (1) (first, second, and third term), the differential correction method was used. Applying this equation to the times of minima given in Table 6 and using a least-squares solution we obtained

$$
\begin{aligned}
\text { Min I }= & 2452244.6102(6)+0.4414340(2) \times E \\
& +1.46(8) \times 10^{-9} \times E^{2} .
\end{aligned}
$$

The variations of $\Delta T$ are displayed in Fig. 7. The observed $\mathrm{O}-\mathrm{C}$ values in this figure are obtained with linear elements. The dashed line in Fig. 7a represents the secular increase in the orbital period of the binary. Figure $7 \mathrm{~b}$ shows the residuals from the parabolic variation. Using Eq. (5) a period change of $\mathrm{d} P / \mathrm{d} t=2.4 \times 10^{-6} \mathrm{~d} \mathrm{yr}^{-1}$ is estimated. Such a value for the period variation is higher than expected for semi-detached or contact binary systems. We need additional times of minima than available to us now in order to determine the mass transfer rate accurately.

\subsubsection{The blue straggler detached binary ES Cnc (S1082)}

We collected all the available minima times of ES Cnc and listed them in Table 7. Using these minima times and the first two terms in Eq. (1) we obtained the new ephemeris of the system.

Min I = HJD 2444 643.2516(42) $+1^{\mathrm{d}} .067797(1) \times E$.

Unlike AH Cnc and EV Cnc, ES Cnc is a detached binary system with no mass flow between the components. However, it may lose mass via a stellar wind. The mass loss from the system can affect the $\mathrm{O}-\mathrm{C}$ diagram, but this non-conservative effect is less effective than the effect of conservative mass transfer. The $\mathrm{O}-\mathrm{C}$ variation of the system, after the corrections of $T_{\mathrm{o}}$ and $P$, is shown in Fig. 8. Spectral studies of the system show the presence of a third body with a $3.3 \mathrm{yr}$ period. To model the light-time effect, we need more times of minima than those we obtained on successive nights.

\subsection{Light curve modeling of close binaries}

\subsubsection{AH Cnc}

In contrast to previous studies, we obtained a 28 day coverage of the high-quality data, including four colour light curves, of
Table 4. Times of the primary and secondary minima in $\mathrm{JD}_{\text {hel }}$ (HJD-

\begin{tabular}{|c|c|c|c|c|c|}
\hline HJD & Meth. & Ref. & HJD & Meth. & Ref. \\
\hline 33626.364 & pg & 1 & 51939.1411 & ccd & 12 \\
\hline 34421.307 & pg & 1 & 51940.2221 & ced & 12 \\
\hline 35219.318 & pg & 1 & 51940.2278 & $\mathrm{ccd}$ & 12 \\
\hline 36656.352 & pg & 1 & 51956.9876 & ced & 13 \\
\hline 37378.322 & pg & 2 & 51957.1642 & $\operatorname{ccd}$ & 13 \\
\hline 37699.300 & pg & 2 & 51958.0662 & $\operatorname{ccd}$ & 13 \\
\hline 38820.7850 & pe & 3 & 51958.2465 & $\operatorname{ccd}$ & 13 \\
\hline 39964.300 & $\mathrm{pg}$ & 1 & 51959.1478 & $\operatorname{ccd}$ & 13 \\
\hline 40329.398 & $\mathrm{pg}$ & 1 & 52314.1944 & $\operatorname{ccd}$ & 12 \\
\hline 40570.9104 & pe & 4 & 52314.2001 & ced & 13 \\
\hline 40678.3230 & pe & 5 & 52315.0943 & $\operatorname{ccd}$ & 12 \\
\hline 41396.332 & pg & 5 & 52315.2771 & $\operatorname{ccd}$ & 12 \\
\hline 41740.908 & pg & 6 & 52995.2842 & ced & 13 \\
\hline 41742.8900 & pe & 6 & 52996.3640 & $\operatorname{ccd}$ & 13 \\
\hline 41752.8040 & pe & 6 & 52997.2642 & ced & 13 \\
\hline 41797.6750 & pe & 6 & 53001.2290 & $\operatorname{ccd}$ & 13 \\
\hline 41815.6980 & pe & 6 & 53004.1135 & ccd & 13 \\
\hline 42537.3000 & pe & 6 & 53004.2951 & $\operatorname{ccd}$ & 13 \\
\hline 43163.554 & pg & 5 & 53005.1948 & $\operatorname{ccd}$ & 13 \\
\hline 43192.214 & pg & 5 & 53005.3747 & $\operatorname{ccd}$ & 13 \\
\hline 43256.371 & pg & 5 & 53006.2758 & ccd & 13 \\
\hline 43931.492 & pg & 5 & 53007.1818 & ccd & 13 \\
\hline 44015.288 & pg & 5 & 53008.2609 & ccd & 13 \\
\hline 47200.6990 & $\operatorname{ccd}$ & 7 & 53009.3435 & $\operatorname{ccd}$ & 13 \\
\hline 47200.8790 & ced & 7 & 53047.3702 & $\operatorname{ccd}$ & 14 \\
\hline 47203.7620 & ccd & 7 & 53083.0540 & ccd & 12 \\
\hline 47203.9440 & ced & 7 & 53084.1348 & $\operatorname{ccd}$ & 12 \\
\hline 50904.288 & pg & 8 & 53426.3926 & ced & 12 \\
\hline 51159.3059 & $\operatorname{ced}$ & 9 & 53426.3931 & $\operatorname{ccd}$ & 14 \\
\hline 51177.1469 & $\mathrm{ccd}$ & 9 & 53437.7466 & ccd & 15 \\
\hline 51177.3275 & $\mathrm{ced}$ & 9 & 53439.1876 & ced & 12 \\
\hline 51179.3103 & $\operatorname{ccd}$ & 9 & 53442.7929 & $\operatorname{ccd}$ & 16 \\
\hline 51229.7727 & $\mathrm{ccd}$ & 10 & 53471.0869 & ccd & 12 \\
\hline 51231.7490 & $\operatorname{ccd}$ & 10 & 53489.2928 & ced & 14 \\
\hline 51245.8122 & $\operatorname{ced}$ & 10 & 53683.5814 & $\operatorname{ccd}$ & 14 \\
\hline 51250.6798 & $\operatorname{ced}$ & 10 & 53750.6235 & $\operatorname{ccd}$ & 14 \\
\hline 51273.0268 & $\operatorname{ccd}$ & 9 & 53765.3982 & $\operatorname{ccd}$ & 17 \\
\hline 51585.1847 & $\operatorname{ccd}$ & 11 & 54060.9837 & ced & 18 \\
\hline
\end{tabular}
2400000 ) for AH Cnc.

1- Kurochkin (1979), 2- Kurochkin (1965), 3- Eggen (1967), 4- Millis (1972), 5- Kurochkin (1970), 6- Whelan et al. (1979), 7- Estimated from light curves of Gilliland et al. (1991), 8- Diethelm (1998), 9- Youn et al. (2003), 10- Blake (2002), 11- Csizmadia et al. (2002), 12- Qian et al. (2006), 13- Zhang et al. (2005), 14- Krajci (2005), 15- Krajci (2006), 16- Nelson (2006), 17- Biro et al. (2006), 18- Nelson (2007).

the overcontact binary system AH Cnc. The multi-colour solution of the system allows us to determine more accurate orbital and physical parameters of the binary than the earlier studies. Figure 2 shows that the system has a total eclipse, which is an important tool to determine the mass ratio accurately in the absence of a good quality spectrum.

The light curve analysis of the system has been made by using the WD code in Mode 3 (Wilson \& Devinney 1971; Wilson 1994). The adopted values are: $T_{1}=6300 \mathrm{~K}$ from the $B-V$ colour index, gravity darkening coefficients, and albedos were chosen as $g_{1}=g_{2}=0.32$ (Lucy 1967) and $A_{1}=A_{2}=0.5$ (Rucinski 1969) and the logarithmic limb-darkening coefficients $\left(x_{1}, x_{2}\right)$ were obtained from van Hamme (1993). The inclination $i$, temperature of the secondary component $T_{2}$, luminosities of the primary component $L_{1}(V, R$, and $I)$, the potential of the common surface $\Omega$, and mass ratio were adjustable parameters. 

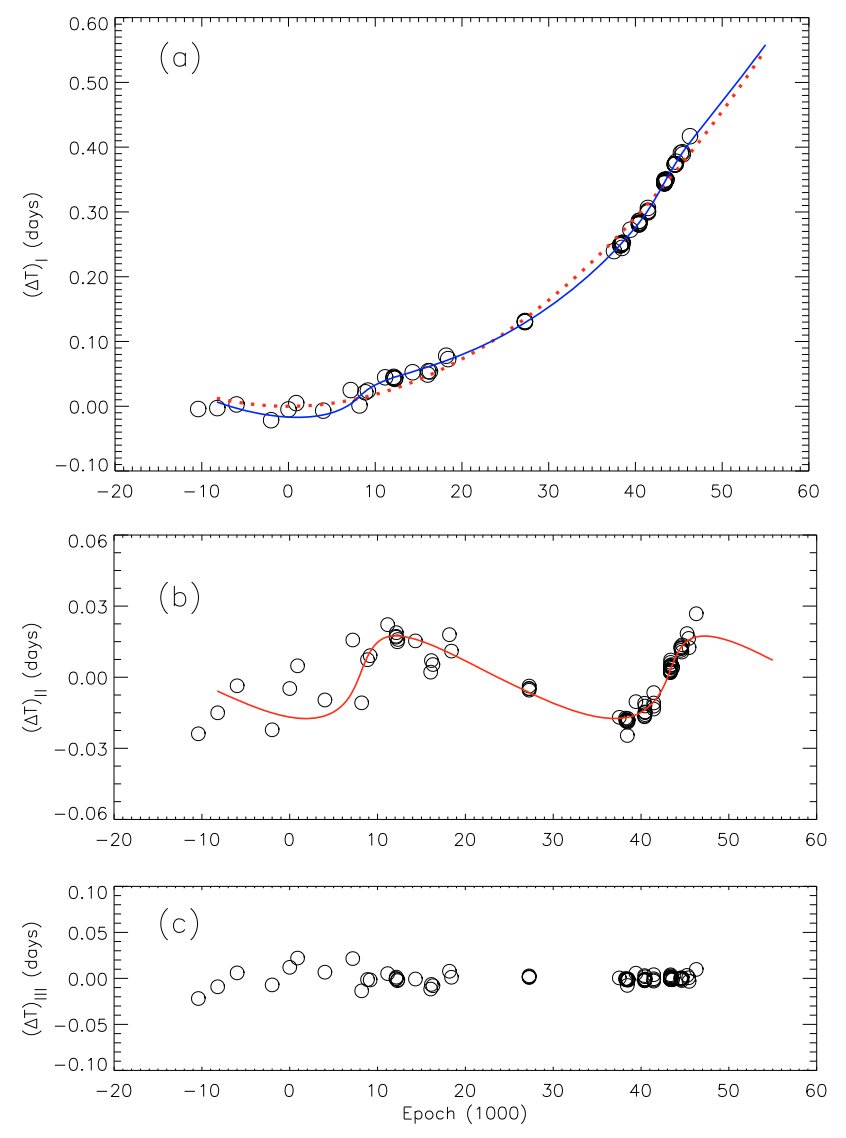

Fig. 6. a) The $(\Delta T)_{I}$ diagram of the times of mid-eclipses for AH Cnc, b) $(\Delta T)_{\text {II }}$ variation of the system after parabolic variation removed, and c) final residual.

$V, R$, and $I$ light curves were analysed simultaneously. The results obtained after several dozen iterations are given in Table 8.

We first modelled the May LC and deduced the parameters. Then we modelled the Jan LC of the system assuming the same orbital parameters and adding free spot and third light parameters. The computed light curves (solid lines) with the parameters given in the last column of Table 8 were compared with all observed light curves shown in Fig. 2. During the solution we consider the deep minimum as the one of the primary. The synthetic light curves were created with the LC program. The filling factor, $f=0.56$, is given by $\left(\Omega_{\text {in }}-\Omega\right) /\left(\Omega_{\text {in }}-\Omega_{\text {out }}\right)$ and varies from 0 to unity from the inner to the outer critical surface. This solution indicates that the system has a deep degree of contact.

The light curve of the system was modelled (see Table 8 ) by Whelan et al. (1979), Sandquist \& Shetrone (2003), Youn et al. (2003), Zhang et al. (2005), Qian et al. (2006), Yakut \& Aerts (2006), and recently by Pribula et al. (2008) using one colour MOST space photometry. In Table 8 they are shown as W, SS, Y, ZZD, YA, Q1- Qian et al. (2006) solution without third body, Q2- Qian et al. solution with third body, P, PS1- present solution (January) with third light, and PS2-present solution (May) with third light, respectively. In the present study, three colour solutions are made and lead to more accurate parameters.

\subsubsection{EV Cnc}

The light curve of EV Cnc is quite different from those of normal contact or near-contact systems. Therefore, different
Table 5. Orbital elements of the tertiary component in AH Cnc.

\begin{tabular}{llll}
\hline \hline Parameter & Unit & Value & Error \\
\hline$T_{o}$ & [HJD] & 2437378.3295 & \pm 0.0012 \\
$P_{o}$ & [day] & 0.3604360 & \pm 0.0000001 \\
$P^{\prime}$ & [year] & 34.7 & \pm 0.2 \\
$T^{\prime}$ & [HJD] & 40374 & \pm 87 \\
$e^{\prime}$ & & 0.68 & \pm 0.03 \\
$\omega^{\prime}$ & $\left.{ }^{\circ}\right]$ & 9.9 & \pm 2.4 \\
$a_{12} \sin i^{\prime}$ & {$[\mathrm{AU}]$} & 4.1 & \pm 0.1 \\
$f(m)$ & {$\left[M_{\odot}\right]$} & 0.056 & \pm 0.005 \\
$m_{3 ; i^{\prime}=30^{\circ}}$ & {$\left[M_{\odot}\right]$} & 1.57 & \\
$m_{3 ; i^{\prime}=90^{\circ}}$ & {$\left[M_{\odot}\right]$} & 0.60 & \\
$Q$ & {$[\mathrm{c} / \mathrm{d}]$} & $1.82 \times 10^{-10}$ & $0.02 \times 10^{-10}$ \\
\hline
\end{tabular}

possibilities were taken into consideration. The minima are quite different, which may be an indication of a semi-detached configuration rather than a contact one, as proposed in the literature. The O'Connell effect is apparent in the light curves of EV Cnc presented in the literature. This effect can be explained by a mass transfer in a semi-detached system that gives rise to a hot region, rather than a spot activity on the surface of low-temperature contact binaries.

The first eleven cosine Fourier coefficients of our light curves of EV Cnc were calculated. Rucincki (1993) and (Selam 2004) have plotted the values of $a_{4}$ versus $a_{2}$ for different contact degrees. In their figure the system EV Cnc lies outside of the contact region. In addition, we have tested our $a_{2}$ and $a_{4}$ values in the Paczynski et al. (2006) diagram, where they plotted about 11000 close binaries. The system is situated close to the semi-detached binaries rather than the contacts. We used Mode 3, Mode 4, and Mode 5 of the WD code (see Table 9) and we tested our results using Phoebe (Prša \& Zwitter 2005). Mode 5 in the DC code was used for the final light curve modeling. This mode solves the light curves of semi-detached binaries, where the secondary component fills its corresponding Roche lobe while the primary is detached. The following parameters were fixed for the light curve analysis: the mean temperature of the hotter component is $T_{\mathrm{h}}=6900 \mathrm{~K}$ according to the colour index and the logarithmic limb darkening coefficients. The adjustable parameters are the inclination, the mass ratio, the temperature of the secondary component, the luminosities, the surface potential, the values of the gravity darkening coefficients $g_{1}$ and $g_{2}$, and the values of the albedos.

The results of a simultaneous three colour light curve solution are summarized in Table 9 . The results of different model solutions (M) have been denoted with different letters. We have assigned Model A for the contact configuration, Model B for the non-thermal contact, and finally Model $\mathrm{C}$ for the semidetached configuration. Our analysis shows that the configuration of EV Cnc is almost contact. The model results (Model C, solid line) and observations are compared in Fig. 3a. The asymmetries in the light curves indicate that the components may have hot or cold activity regions on their surfaces. Hence, throughout the solutions the spotted model assumption was considered. A hot spot and two cold ones determined on the surfaces of the cooler component. The spot parameters given in Table 9 are common for all the three models.

\subsubsection{ES Cnc}

ES Cnc is an RS CVn type active close binary. The system is brighter than AH Cnc and EV Cnc; therefore, RTT150 
Table 6. Times of the primary (I) and secondary (II) minima in $\mathrm{JD}_{\text {hel }}$ (HJD- 2400000$)$ for EV Cnc.

\begin{tabular}{lrrll}
\hline HJD & Epoch & $\Delta T_{\mathrm{I}}$ & $\Delta T_{\mathrm{II}}$ & Ref. \\
\hline 51229.7723 & -2299 & 0.0175 & 0.0098 & 1 \\
51231.7441 & -2294.5 & 0.0028 & -0.0048 & 1 \\
51250.7368 & -2251.5 & 0.0139 & 0.0065 & 1 \\
51603.2028 & -1453 & -0.0047 & -0.0077 & 2 \\
51604.0883 & -1451 & -0.0020 & -0.0051 & 2 \\
52244.6052 & 0 & -0.0050 & -0.0050 & 3 \\
53108.2776 & 1956.5 & 0.0030 & -0.0026 & 4 \\
53425.4532 & 2675 & 0.0087 & -0.0018 & 5 \\
53426.3392 & 2677 & 0.0118 & 0.0013 & 5 \\
53443.3450 & 2715.5 & 0.0224 & 0.0117 & 6 \\
53682.6029 & 3257.5 & 0.0234 & 0.0079 & 5 \\
54144.1280 & 4303 & 0.0299 & 0.0029 & 7 \\
54147.2024 & 4310 & 0.0142 & -0.0128 & 7 \\
54147.6584 & 4311 & 0.0288 & 0.0017 & 7 \\
54150.3054 & 4317 & 0.0272 & 0.0001 & 7 \\
54151.6301 & 4320 & 0.0276 & 0.0004 & 7 \\
54152.0721 & 4321 & 0.0282 & 0.0010 & 7 \\
54152.5075 & 4322 & 0.0221 & -0.0051 & 7 \\
\hline
\end{tabular}

1- Blake (2002); 2- Csizmadia et al. (2002); 3- Csizmadia et al. (2006); 4- Krajci (2005); 5- This study; 6- Hübscher et al. (2005); 7- Pribulla et al. (2008).

observations were saturated. The radial velocity curve of the system has been obtained by van den Berg et al. (2001) and Sandquist et al. (2003). Full light curves of the system were obtained with the Mercator telescope. The light variations of the system show small variations on a time scale of weeks.

We have calculated the Fourier coefficients of the light curve. It falls in the detached region on the $a_{4}$ vs. $a_{2}$ plane. The light curve of ES Cnc has been well analyzed before by van den Berg et al. (2001) using the Eclipsing Light Curve code of Orosz \& Hauschildt (2000), by Sandquist et al. (2003) with Nightfall and by Pribulla et al. (2008). All researchers solved the system assuming the third light effect and stellar spots. We used the WD code under the assumptions of the presence of a third light and spots. The adopted values are: $T_{1}=7325 \mathrm{~K}$, gravity darkening coefficients, and albedos were chosen as $g_{1}=1, g_{2}=0.32$ and $A_{1}=1, A_{2}=0.5$ and the logarithmic limb-darkening coefficients $\left(x_{1}, x_{2}\right)$ were obtained from van Hamme (1993). The semi major axis of the relative orbit, $a$; binary center-of-mass radial velocity, $V_{\gamma}$; inclination, $i$; temperature of the secondary component, $T_{2}$; luminosities of the primary component, $L_{1}$; the potentials of the components, $\Omega_{1}$ and $\Omega_{2}$, and spot parameters (latitude, longitude, size, and temperature factor) were adjustable parameters. The results of a simultaneous solution of LC and RV are given in Table 10. The comparison of the observations and model are shown in Fig. 9.

\subsection{Frequency analysis of the $\delta$ Scuti stars EX Cnc (S1284) and EW Cnc (S1280)}

For both stars, we carried out a frequency analysis and a mode identification based on the multi-colour data that are available. Bruntt et al. (2007), hereafter referred to as B07, have already conducted a successful multi-site campaign on EX $\mathrm{Cnc}$ and EW Cnc. Their data provided 26 frequencies for EX $\mathrm{Cnc}$ and 41 for EW Cnc, but they could not carry out a photometric mode identification due to the fact that their measurements were acquired in only one filter. Therefore they were not able to
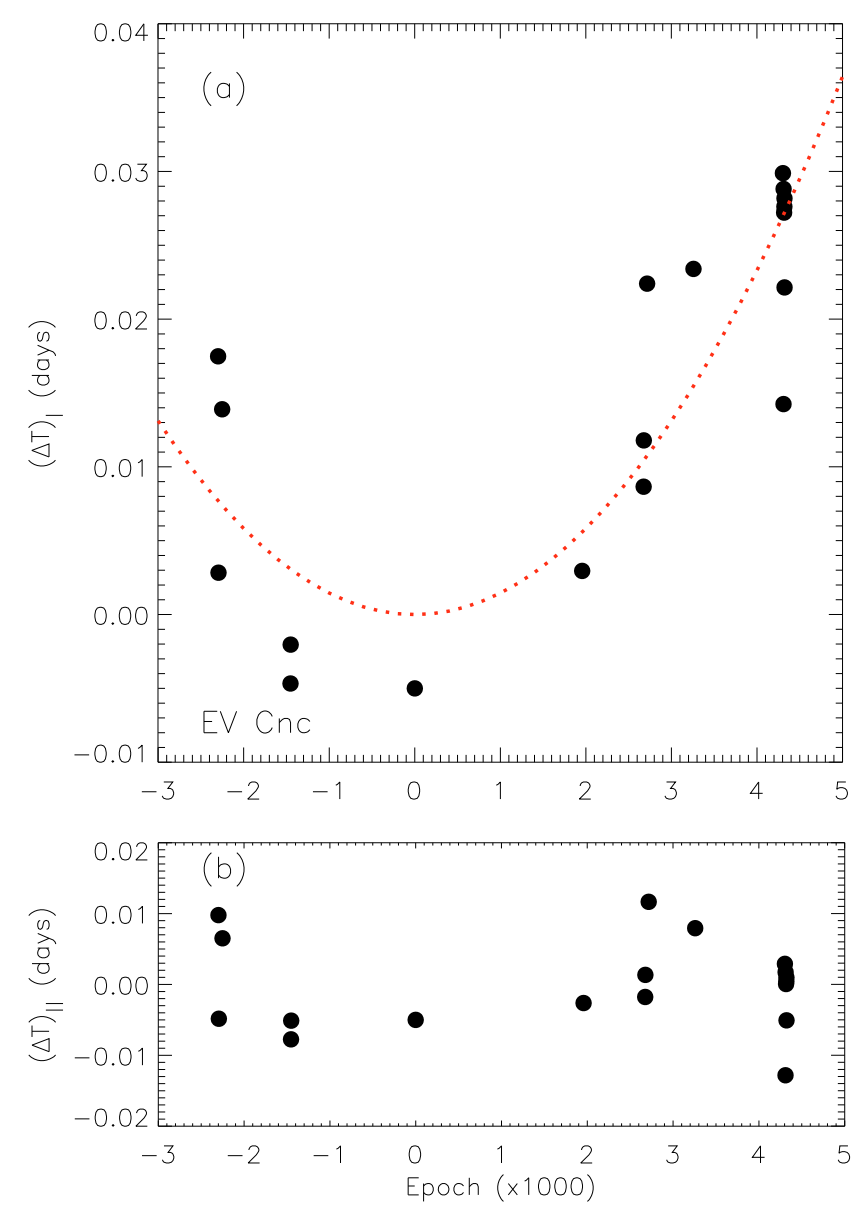

Fig. 7. The $(\Delta T)_{I}$ diagram of the times of mid-eclipses for EV Cnc.

constrain fundamental parameters of the stars and to provide a unique seismic model.

Our data consist of multi-colour data taken simultaneously in the $V, R$, and $I$ filters at the Mercator telescope. In contrast to B07, our observations are single-site data which complicates the search for pulsation frequencies due to daily aliasing problems. Our aim was to use the frequency values that were determined by B07 and to carry out a mode identification using the method of amplitude ratios and phase differences (Dupret et al. 2003; Daszynska-Daszkiewicz et al. 2003).

To determine differential magnitudes of the stars in the different filters, we had six different comparison stars on the CCD-frames at our disposal. Most of these comparisons proved to show systematic trends on the time scales of days. These trends are smallest for the comparisons $\mathrm{C} 3$ and $\mathrm{C} 5$, which we therefore used to determine differential magnitudes. For our search for pulsation frequencies we used Fourier techniques for non-equidistant measurements (discrete Fourier transformation) and multi-periodic least-squares fitting techniques for sinusoidal variations. The frequency search was carried out in the following iterative manner. We selected the highest frequency peak in the Fourier spectrum, compute a least-squares fit of this peak and all previously found frequencies by improving frequencies, amplitudes and phases of a sum of sinusoids. In case of aliasing, we selected the frequency value that resulted in the lowest residuals after pre-whitening. We then pre-whiten the original data with the determined least-squares fit and computed a Fourier spectrum of the residuals. This procedure was carried out iteratively 
Table 7. Times of the primary (I) and secondary (II) minima in $\mathrm{JD}_{\text {hel }}$ (HJD- 2400000 ) for ES Cnc.

\begin{tabular}{lrrll}
\hline \hline HJD & Epoch & $\Delta T_{I}$ & $\Delta T_{I I}$ & Ref. \\
\hline 43191.036 & -1360 & 0.0472 & -0.0118 & 1 \\
44643.250 & 0 & 0.0478 & -0.0016 & 1 \\
45325.586 & 639 & 0.0570 & 0.0122 & 1 \\
46773.492 & 1995 & 0.0208 & -0.0144 & 1 \\
47861.605 & 3014 & 0.0415 & 0.0136 & 1 \\
47920.333 & 3069 & 0.0403 & 0.0127 & 1 \\
47944.869 & 3092 & 0.0168 & -0.0106 & 1 \\
51218.744 & 6158 & 0.0048 & -0.0009 & 1 \\
51539.078 & 6458 & -0.0024 & -0.0059 & 1 \\
51602.0961 & 6517 & 0.0152 & 0.0122 & 2 \\
51603.1655 & 6518 & 0.0168 & 0.0138 & 2 \\
53765.4321 & 8543 & -0.0197 & -0.0083 & 3 \\
54165.8639 & 8918 & -0.0144 & -0.0004 & 4 \\
54167.9957 & 8920 & -0.0182 & -0.0042 & 4 \\
54170.1339 & 8922 & -0.0156 & -0.0016 & 4 \\
54171.2019 & 8923 & -0.0154 & -0.0014 & 4 \\
54172.2696 & 8924 & -0.0155 & -0.0015 & 4 \\
54173.3370 & 8925 & -0.0159 & -0.0019 & 4 \\
\hline
\end{tabular}

1- van den Berg et al. (2001), 2- Csizmadia et al. (2002), 3- Biro et al. (2006), 4- Pribulla et al. (2008).

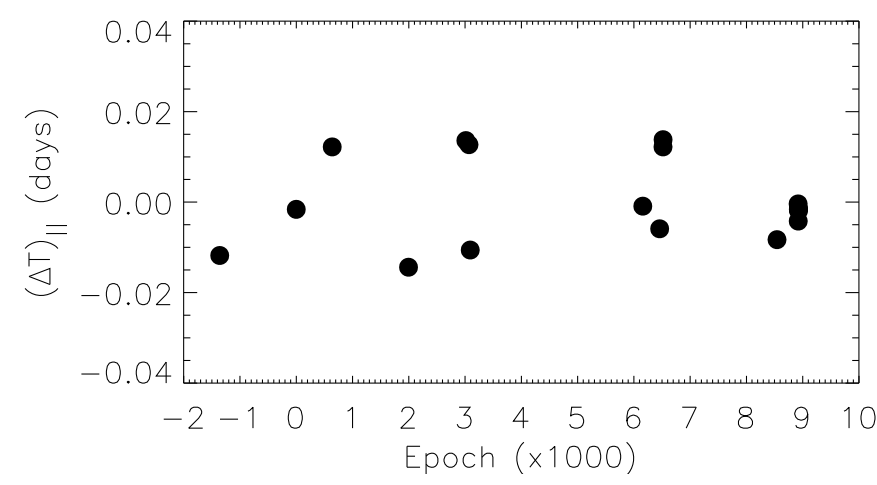

Fig. 8. The $(\Delta T)_{\text {II }}$ diagram of the times of mid-eclipses for ES Cnc.

until the amplitude signal-to-noise ratio (SNR) of the highest frequency peak remained below a value of 4.0 (Breger 1999). We used the software package FAMIAS (Zima 2008) for the frequency search as well as for the mode identification.

\subsubsection{EX Cnc}

We used the differential light curve $E X-C 5$ in $V$ of EX Cnc and C5 for our frequency analysis since these data showed the best long term stability. The highest peak is at $f_{1}=20.61 \mathrm{c} / \mathrm{d}$ which corresponds to the frequency $g_{2}=20.63 \mathrm{c} / \mathrm{d}$ detected by B07. The highest peak detected by B07 is at $g_{1}=19.61 \mathrm{c} / \mathrm{d}$, which is exactly $1 \mathrm{c} / \mathrm{d}$ separated from $f_{1}$. Due to the strong daily aliasing in our data, we cannot exclude that $19.6 \mathrm{c} / \mathrm{d}$ is the correct frequency value. We tested both scenarios and preferred the frequency solution with $20.61 \mathrm{c} / \mathrm{d}$ due to the lower residuals after pre-whitening with the least-squares fit. The residuals show no significant frequency peaks. We also searched in the residuals for frequencies with a $S N R<4$ that were reported by B07, but could not find any acceptable agreement. The amplitudes, phases, and the SNR of $f_{1}$ in the different filters are displayed in Table 11 . The phase is indicated in units of $2 \pi$ and computed relative to the zero point at HJD 2450000 . The uncertainty of the measurements in units of the last digit is indicated in brackets. The noise for the SNR calculation was computed from the mean of the amplitude of the pre-whitened Fourier spectrum in a $3 \mathrm{c} / \mathrm{d}$ box around each frequency.

The main criterion for the mode identification of $\delta$ Sct stars is the phase difference in different photometric passbands. The phase difference can be used to distinguish between different values of the degree $\ell$. As can be seen in Table 11, the uncertainty of the phase values is of the same order as the difference between the three filters. We therefore conclude that the quality of the data is insufficient to acquire a conclusive mode identification for this frequency.

\subsubsection{EW Cnc}

We chose the comparison star C3 for this object due to the best long term stability of the differential light curve. The two highest peaks in our data at $f_{1}=18.82$ and $f_{2}=20.19 \mathrm{c} / \mathrm{d}$ and with amplitudes of about 3 and 2 mmag, respectively, in V agree well with the frequencies of highest amplitude detected by B07. We find an additional frequency at $f_{3}=37.60 \mathrm{c} / \mathrm{d}$, whereas B07 report two frequencies very closeby, at 37.617 and 37.654. Although, the theoretical frequency resolution of our data is $0.01 \mathrm{c} / \mathrm{d}$ (Loumos \& Deeming 1978) - enough to separate the two frequencies, no frequency peak remains close to these values after pre-whitening with $f_{3}$. We find two more frequencies that were reported by B07, namely at $f_{4}=31.43$ and $f_{5}=27.86 \mathrm{c} / \mathrm{d}$. After pre-whitening with the five frequencies, a significant peak that was not found by B07 with a SNR of 6.5 at $f_{6}=37.13 \mathrm{c} / \mathrm{d}$ remains. The residuals after pre-whitening show no significant peaks and no additional frequencies can be identified with frequencies reported by B07. The Cousins $R$ and $I$ light curves are much noisier than the $V$ data. Therefore, much less frequencies could be detected in these filters. We relaxed the SNR criterion for significance to $S N R \geq 3.5$ for the frequencies that were already detected in the $V$-band. Table 12 reports the result of the frequency search for the light curves of EW Cen.

Also for the derived pulsation phases of EW Cnc, the formal statistical uncertainties that were derived from the least-squares fitting, are of the same order as the phase difference between the different filters. We therefore, unfortunately, have to conclude that also for this star the quality and amount of data are not sufficient for a successful photometric mode identification.

\section{Results and conclusions}

We presented 28 days long term multi-colour observations for the solar age galactic open cluster M 67.

Radial velocity studies of EV Cnc and $\mathrm{AH}$ Cnc made by Blake (2002) and Whelan et al. (1979), respectively. Because of their faintness and configuration, the parameters were not as accurate as those of ES Cnc. It is useful, therefore, to deduce the mass ratio from a LC analysis. AH Cnc is an overcontact binary showing total eclipses. A multi-colour analysis was made. The inclination angle of the system was determined $88^{\circ}$ and the mass ratio of the components was estimated 0.17 . We have collected 70 minima times from literature and did a period analysis. The O-C diagram of AH Cnc show shallow sine-like variation superimposed on a parabolic variation. The mass transfer rate is estimated to be $\mathrm{d} M / \mathrm{d} t=+9.4 \times 10^{-8} M_{\odot} \mathrm{yr}^{-1}$. Using the brightness of the systems (Table 2) their distance modules, and bolometric corrections we deduced the absolute physical parameters of the components of AH Cnc (Table 13). For the solar values, 
Table 8. Photometric elements of the over contact binary AH Cnc. See text for the details.

\begin{tabular}{|c|c|c|c|c|c|c|c|c|c|c|}
\hline Parameter & $\overline{W W}$ & $\overline{S S}$ & $\overline{Y Y}$ & $\overline{Z Z D}$ & $\overline{Y Y A}$ & $\overline{Q 1}$ & $\overline{Q 2}$ & $\overline{P P}$ & $P S 1$ & $P S 2$ \\
\hline \multicolumn{11}{|l|}{ Geometric parameters: } \\
\hline$i\left(^{\circ}\right)$ & 65 & 86 & 86 & 82.8 & 87.6 & 90.0 & 90.0 & 89.5 & 88.2 & $88.2(7)$ \\
\hline$\Omega_{1,2}$ & - & - & 2.078 & 2.037 & 2.008 & 2.130 & 2.088 & 2.023 & 2.093 & $2.093(3)$ \\
\hline$q$ & 0.75 & 0.157 & 0.159 & 0.149 & 0.144 & 0.160 & 0.168 & 0.130 & 0.168 & $0.168(2)$ \\
\hline$f(\%)$ & 85 & 70 & 49 & 65 & 87 & 37 & 59 & & 56 & 56 \\
\hline \multicolumn{11}{|l|}{ Radiative parameters: } \\
\hline$T_{1}(\mathrm{~K})$ & & & 6300 & & 6300 & 6300 & 6300 & 6300 & 6300 & 6300 \\
\hline$T_{2}(\mathrm{~K})$ & & & 6100 & 6354 & 6525 & 6179 & 6265 & 6368 & 6275 & $6275(90)$ \\
\hline Albedo $*\left(A_{1}=A_{2}\right)$ & & & 0.5 & & 0.5 & 0.5 & 0.5 & 0.5 & 0.5 & 0.5 \\
\hline \multicolumn{11}{|l|}{$\begin{array}{l}\text { Gravity brightening* }\left(g_{1}=g_{2}\right) \\
\left(\frac{l_{1}}{L_{2}}\right):\end{array}$} \\
\hline$\left(l_{\text {total }}\right)$ & & & 0.850 & 0.830 & & 0.844 & 0.819 & & 0.790 & 0.783 \\
\hline$R$ & & & & & 0.827 & & & & 0.792 & 0.780 \\
\hline$I$ & & & & & & & & & 0.795 & 0.783 \\
\hline$\left(l_{\text {total }}\right) \cdot$ & & & & & & & 0.007 & & 0.034 & 0.036 \\
\hline$R$ & & & & & & & & & 0.031 & 0.040 \\
\hline$I$ & & & & & & & & & 0.026 & 0.035 \\
\hline \multicolumn{11}{|l|}{ Fractional radii } \\
\hline $\bar{r}_{1}$ & & & 0.561 & 0.572 & 0.586 & 0.5555 & 0.5603 & & 0.5584 & $0.5584(14)$ \\
\hline $\bar{r}_{2}$ & & & 0.258 & 0.260 & 0.271 & 0.2505 & 0.2658 & & 0.2531 & $0.2630(60)$ \\
\hline \multicolumn{11}{|l|}{ Spot parameters: } \\
\hline Colatitude $\left(^{\circ}\right)$ & & & & & & & & & $113(5)$ & $98(6)$ \\
\hline Longitude $\left(^{\circ}\right)$ & & & & & & & & & $121(7)$ & $105(9)$ \\
\hline Spot radius $\left({ }^{\circ}\right)$ & & & & & & & & & $35(2)$ & $24(3)$ \\
\hline Spot temperature $\left(T_{\text {spot }} / T_{\text {star }}\right)$ & & & & & & & & & $0.91(1)$ & $0.92(1)$ \\
\hline rms & & & & & & & & & 0.018 & 0.031 \\
\hline
\end{tabular}

Table 9. Photometric elements of EV Cnc.

\begin{tabular}{lccl}
\hline \hline Parameter & Model A & Model B & Model C \\
\hline$i\left({ }^{\circ}\right)$ & 46.7 & 48.6 & $41.8(5)$ \\
$\Omega_{1}$ & 3.30 & 3.133 & 2.709 \\
$q$ & 0.69 & 0.59 & $0.40(1)$ \\
$T_{\mathrm{h}}(\mathrm{K})$ & 6900 & 6900 & 6900 \\
$T_{\mathrm{c}}(\mathrm{K})$ & 5630 & 5830 & $5200(165)$ \\
$\left(\frac{L_{\mathrm{c}}}{L_{\mathrm{c}} L_{\mathrm{h}}}\right)_{V}$ & 0.92 & & 0.382 \\
$\left(\frac{L_{\mathrm{c}}}{L_{\mathrm{c}}+L_{\mathrm{h}}}\right)_{R}$ & 0.89 & & 0.443 \\
$\left(\frac{L_{\mathrm{c}}}{L_{\mathrm{c}}+L_{\mathrm{h}}}\right)_{I}$ & 0.86 & & 0.484 \\
$\bar{r}_{\mathrm{c}}$ & 0.40 & 0.38 & $0.452(2)$ \\
$\bar{r}_{\mathrm{h}}$ & 0.33 & 0.29 & $0.300(3)$ \\
Spot parameters : & & & \\
three spots on secondary: & & & \\
Colatitude $\left({ }^{\circ}\right)$ & & & $49(3), 119(6), 87(4)$ \\
Longitude $\left({ }^{\circ}\right)$ & & & $203(10), 177(12), 11(3)$ \\
Spot radius $\left({ }^{\circ}\right)$ & & & $21(3), 28(4), 16(2)$ \\
Spot temperature $T_{\text {spot }} / T_{\text {star }}$ & & & $0.89(3), 0.88(2), 1.15(2)$ \\
rms & & & 0.012 \\
\hline
\end{tabular}

we have taken $T_{\mathrm{e}}=5780 \mathrm{~K}, M_{\text {bol }}=4.75$, and we adopted $E(B-V)=41 \mathrm{mmag}$ for M 67 (Taylor 2007). The mass of the primary star of AH Cnc has been estimated from the mass-radius relation of well-known LTCBs (Yakut 2005, 2006) and we obtained a value of $1.33 M_{\odot}$. Zhang et al. (2005), using the distance modulus, reported a mass of $1.20 M_{\odot}$ while Qian et al. (2006) found $1.1 M_{\odot}$ from the use of a mass-period relation. These three values are consistent within the uncertainties.

The light curves of EV Cnc and ES Cnc show asymmetries in their maxima. These can be due to stellar activity on the surface of the stars. We have proven that EV $\mathrm{Cnc}$ is a nearly contact binary system rather than a detached or an overcontact binary, consistent with Blake's (2002) prediction. For the first time we presented the analysis of the complex light curve of EV Cnc. Different possibilities were considered during the LC solutions. First of all, we computed solutions with different $q$-values [0.30:0.95] and obtained the smallest sigma value at $q=0.5$. The value of $q$ was chosen as adjustable parameter to perform the final solution. The solution indicates a hot spot located on the surface of the star an impact region of flowing material from the secondary star that faces the cooler component. Blake \& Rucinski (2004) reported a similar conclusion from their spectroscopic data. We estimated the period variation rate from the $\mathrm{O}-\mathrm{C}$ diagram to be $2 \times 10^{-6}$ days per year. This rate is higher than the one expected in semi-detached and contact binaries. A reason for this could be the restricted available number of times of minima (spread over only $\sim 7$ years) of the system. New observations of the system are needed to estimate accurate mass accretion rate. We could not decide firmly whether EV Cnc is a contact or a semi-detached binary. The system can also be in a stage of evolution between these two configurations. To obtain more accurate parameters, the mass ratio should be estimated from high quality spectroscopic observations.

We combined all the radial velocities of ES Cnc obtained by van den Berg et al. (2001) and Sandquist et al. (2003) with the LCs obtained in this study. We found $q=0.777$ and $i=68^{\circ} .3$. The solutions indicate that $18 \%$ of the secondary star's surface is covered by cold spots. In addition, the contribution of the third light, - previously spectroscopically determined - at 0.25 phase was estimated to be $42 \%\left(l_{3} / l_{\text {total }}\right)$ from our observations. These results are in good agreement with those of van den Berg et al. (2001) but differ from the values given by Sandquist et al. (2003) and Pribulla et al. (2008). We calculated the masses, radii, luminosities, etc. of the components of ES Cnc (Table 13). 
Table 10. Photometric parameters of detached system ES Cnc.

\begin{tabular}{lllll}
\hline \hline Parameter & van den Berg et al. (2001) & Sandquist et al. (2003) & Pribulla et al. (2008) & Present study \\
\hline$i\left(^{\circ}\right)$ & $64(1)$ & $68(1)$ & 66.2 & $68.3(2.8)$ \\
$\Omega_{1}$ & - & - & 3.532 & $4.12(21)$ \\
$\Omega_{2}$ & - & - & 3.922 & $4.67(36)$ \\
$a\left(R_{\odot}\right)$ & $7.2(4)$ & - & & $6.629(144)$ \\
$q$ & $0.63(4)$ & - & 0.68 & $0.777(16)$ \\
$V_{\gamma}$ & $0.63(4)$ & - & & $11(1)$ \\
$T_{A a}(\mathrm{~K})$ & $6480(25)$ & $7325(50)$ & $7325(50)$ \\
$T_{A b}(\mathrm{~K})$ & $5450(40)$ & $6000(200)$ & 5543 & $5975(200)$ \\
$\left(\frac{l_{1}}{l_{1}+l_{2}}\right)_{V}$ & - & - & & 0.82 \\
$\left(\frac{l_{3}}{l_{\text {total }}}\right)_{V}$ & - & - & 0.68 & $0.42(15)$ \\
$\bar{r}_{1}$ & - & - & & $0.309(21)$ \\
$\bar{r}_{2}$ & - & - & & $0.222(18)$ \\
Spot parameters $($ spots on cool star): & & & & \\
Colatitude $\left({ }^{\circ}\right)$ & & & $179.9,296.6$ & 32,135 \\
Longitude $\left(^{\circ}\right)$ & & & $11.5,12.5$ & 328,57 \\
Spot radius $\left({ }^{\circ}\right)$ & & & $0.80,0.80$ & 33,30 \\
Spot temperature $\left(T_{\text {spot }} / T_{\text {star }}\right)$ & & & & $0.83,0.83$ \\
rms & & & & 0.175 \\
\hline
\end{tabular}
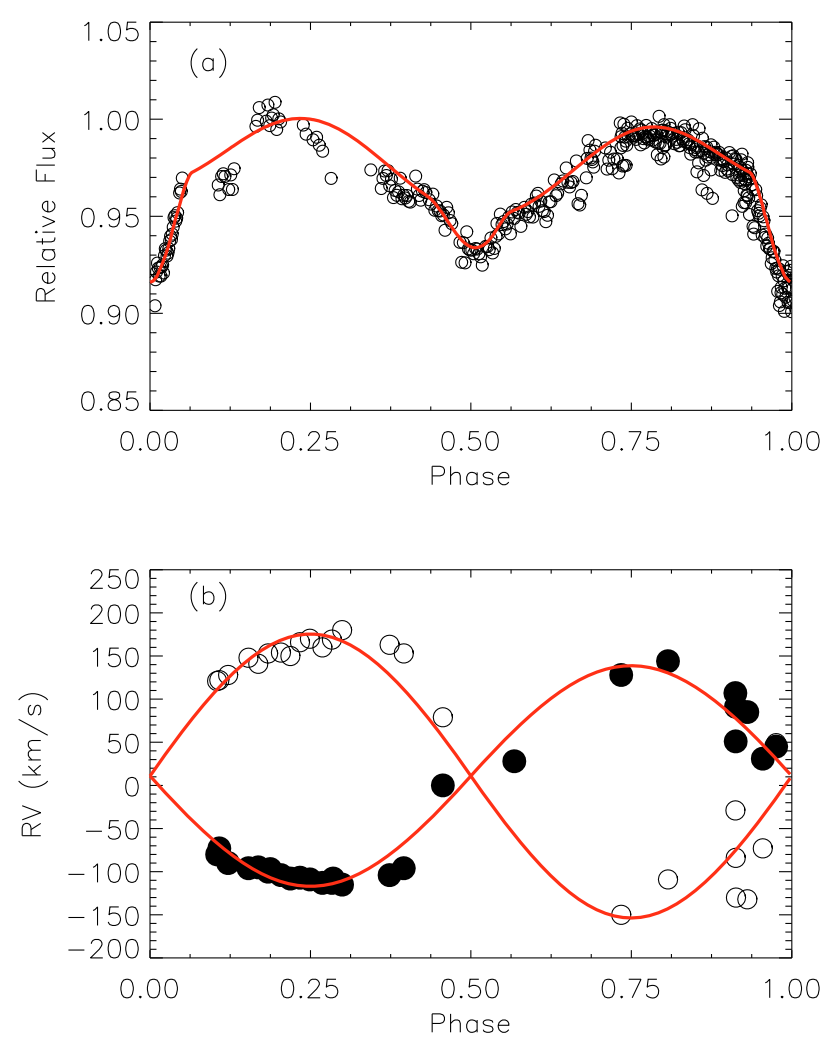

Fig. 9. The observed $V$ band and computed light (a) and radial velocity curves (b) of ES Cnc.

Detached, semi-detached, and contact binaries belonging to a cluster, provide us a precious laboratory to study the evolution of close binary systems. There is an evolutionary connection between late-type contact systems (LTCBs), semi detached (NCBs), and detached (DCBs) systems (Yakut \& Eggleton 2005 and references therein for details). The parameters of the close binaries AH Cnc, EV Cnc, and ES Cnc are compared in Figs. 1-3 of Yakut \& Eggleton (2005) with low-temperature
Table 11. Results of the frequency analysis for EX Cnc.

\begin{tabular}{lllll}
\hline \hline Frequency (c/d) & Filter & $A(\mathrm{mmag})$ & $\phi$ & $S / N$ \\
\hline & $V$ & $5.6(2)$ & $0.408(8)$ & 6.5 \\
$f_{1}=20.605(11)$ & $R$ & $4.2(3)$ & $0.404(11)$ & 4.7 \\
& $I$ & $4.0(3)$ & $0.411(12)$ & 6.5 \\
\hline
\end{tabular}

Table 12. Same as Table 11 but, for EW Cnc.

\begin{tabular}{lllll}
\hline \hline Frequency $(\mathrm{c} / \mathrm{d})$ & Filter & $A(\mathrm{mmag})$ & $\phi$ & $S / N$ \\
\hline \multirow{3}{*}{$f_{1}=18.824(11)$} & $V$ & $3.0(3)$ & $0.912(15)$ & 7.1 \\
& $R$ & $1.7(4)$ & $0.881(31)$ & 3.5 \\
& $I$ & $1.4(4)$ & $0.928(45)$ & 3.5 \\
$f_{2}=20.196(11)$ & $R$ & $2.1(3)$ & $0.097(21)$ & 4.9 \\
& $I$ & $1.5(4)$ & $0.072(35)$ & 3.5 \\
$f_{3}=37.606(11)$ & $V$ & $1.6(4)$ & $0.151(38)$ & 3.9 \\
& $I$ & $2.6(3)$ & $0.237(28)$ & 7.6 \\
$f_{4}=31.438(11)$ & $V$ & $1.6(3)$ & $0.217(27)$ & 3.8 \\
& $R$ & $1.6(4)$ & $0.047(29)$ & 7.0 \\
$f_{5}=27.862(11)$ & $V$ & $1.2(3)$ & $0.067(34)$ & 3.7 \\
$f_{6}=37.135(11)$ & $V$ & $1.4(3)$ & $0.793(32)$ & 3.9 \\
\hline & & & &
\end{tabular}

contact, near-contact, and detached binaries whose physical parameters are well-known. The figures show that they are in a good agreement. The evolution of an individual star in a close binary system is influenced by the nuclear evolution, mass transfer (MT), mass loss (ML) and angular momentum loss (AML). The effect of the AML mechanism depends on the mass of the component, the mass ratio, and the separation of components. Using the obtained physical parameters one can estimate the MT, ML, and AML rates (see Yakut et al. 2008 for details) for AH Cnc, EV Cnc, and ES Cnc.

Light and double-lined radial velocity curves of ES Cnc are available. Using the system parameters given in Table 13, we determined the distance of the system and thus the one of the cluster to be $857(33) \mathrm{pc}$, while the distance modulus is $(m-M)_{V}=$ $9.80(8)$. The distance modulus given previously by Sarajedini et al. (2004) amounts to 9.74(6), while Sandage et al. (2003), 
Table 13. Astrophysical parameters for the close binaries AH Cnc and ES Cnc.

\begin{tabular}{|c|c|c|c|c|c|}
\hline \multirow[b]{2}{*}{ Quantity } & \multirow[b]{2}{*}{ Unit } & \multicolumn{2}{|l|}{ AH Cnc } & \multicolumn{2}{|c|}{ ES Cnc } \\
\hline & & Pri. & Sec. & Pri. & Sec. \\
\hline Mass $(M)$ & $M_{\odot}$ & $1.47(15)$ & $0.25(3)$ & $1.94(13)$ & $1.50(9)$ \\
\hline Radius $(R)$ & $R_{\odot}$ & $1.40(9)$ & $0.68(5)$ & $2.05(7)$ & $1.47(6)$ \\
\hline Effective Temperature $\left(T_{\mathrm{e}}\right)$ & $\mathrm{K}$ & 6300 & $6275(90)$ & $7325(50)$ & $5975(200)$ \\
\hline $\operatorname{Luminosity}(L)$ & $L_{\odot}$ & $2.78(50)$ & $0.64(11)$ & $10.8(7)$ & $2.5(6)$ \\
\hline Surface Gravity $(\log g)$ & $\operatorname{cgs}$ & 4.31 & 4.17 & 4.10 & 4.28 \\
\hline Bolometric Magnitude $\left(M_{\mathrm{bol}}\right)$ & mag & 3.64 & 5.23 & 2.16 & 3.77 \\
\hline Visual Magnitude $(V)$ & mag & 13.60 & 15.19 & 12.05 & 13.80 \\
\hline Absolute Magnitude $\left(M_{V}\right)$ & mag & 3.80 & 5.39 & 2.25 & 3.95 \\
\hline Bolometric Correction (B.C.) & mag & -0.16 & -0.16 & -0.09 & -0.18 \\
\hline Period Change Rate $(\dot{P})$ & $\mathrm{dyr}^{-1}$ & $+3.7 \times 10^{-7}$ & & +5.1 & \\
\hline Mass Transfer Rate $(\dot{M})$ & $M_{\odot} \mathrm{yr}^{-1}$ & $+9.4 \times 10^{-8}$ & & & \\
\hline Distance $(d)$ & $\mathrm{pc}$ & & & & \\
\hline
\end{tabular}

Sandquist (2004), and Grocholski \& Sarajedini (2003) found $9.65,9.72(5)$, and 9.64, respectively. The distance modulus we derived in this work is thus in agreement with those previous studies.

Acknowledgements. We thank the referee for providing constructive comments. K.Y. acknowledges support by the Research Council of the University of Leuven under a DB fellowship and thanks the Turkish Scientific and Technical Research Council (TÜBITAK). W.Z. is supported by the FP6 European Coordination Action HELAS and by Research Council of the University of Leuven under grant GOA/2003/04. B.K. acknowledges support by the Communauté Française de Belgique and Ministry of National Education, Turkey. S.S. is an Apirant Fellow of the Fund for Scientific Research, Flanders (FWO).

\section{References}

Akalin, A., \& Derman, E. 1997, A\&AS, 125, 407

Belloni, T., Verbunt, F., \& Schmitt, J. H. M. M. 1993, A\&A, 269, 175

Belloni, T., Verbunt, F., \& Mathieu, R. D. 1998, A\&A, 339, 431

Biro, I., Borkovits, T., Csizmadia, S., et al. 2006, Inf. Bull. Var. Stars, 5684, 1

Blake, R. M. 2002, Ph.D. Thesis (Canada: York University)

Blake, R. M., \& Rucinski, S. M. 2004, A\&AS, 205, 8504

Breger, M., Pamyatnykh, A. A., Pikall, H., et al. 1999, A\&A, 341, 151

Bruntt, H., Stello, D., Suárez, J. C., et al. 2007, MNRAS, 378, 1371

Carraro, G., Chiosi, C., Bressan, A., et al. 1994, A\&AS, 103, 375

Csizmadia, S., Zhou, A. Y., Konyves, V., Varga, Z., \& Sandor, Z. 2002, Inf. Bull. Var. Stars, 5230, 1

Csizmadia, S., Klagyivik, P., Borkovits, T., et al. 2006, Inf. Bull. Var. Stars, 5736, 1

Daszynska-Daszkiewicz, J., Dziembowski, W. A., \& Pamyatnykh, A. A. 2003, A\&A, 407, 999

Diethelm, R. 1998, BBSAG Bull., 117

Dupret, M.-A., De Ridder, J., De Cat, P., et al. 2003, A\&A, 398, 677

Efremov, Y. N., Kholopov, P. N., Kukarkin, B. V., et al. 1964, Inf. Bull. Var. Stars, 75, 1

Eggen, O. J. 1967, MmRAS, 70, 111

Giampapa, M. S., Hall, J. C., Radick, R. R., \& Baliunas, S. L. 2006, ApJ, 651, 444

Gilliland, R. L., \& Brown, T. M. 1992, AJ, 103, 1945

Gilliland, R. L., Brown, T. M., Duncan, D. K., et al. 1991, AJ, 101, 541

Grocholski, A. J., \& Sarajedini, A. 2003, MNRAS, 345, 1015

Hurley, J. R., Pols, O. R., Aarseth, S. J., et al. 2005, MNRAS, 363, 293

Hübscher, J., Paschke, A., \& Walter, F. 2005, Inf. Bull. Var. Stars, 5657, 1

Irwin, J. B. 1952, ApJ, 116, 211

Jura, M. 1986, ApJ, 309, 732

Kalomeni, B., Yakut, K., Keskin, V., et al. 2007, AJ, 134, 642

Kaluzny, J., \& Radczynska, J. 1991, Inf. Bull. Var. Stars, 3586, 1

Kurochkin, N. E. 1960, Astronomicheskij Tsirkulyar, 212, 9
Kurochkin, N. E. 1965, Inf. Bull. Var. Stars, 79, 1

Kurochkin, N. E. 1979, Astronomicheskij Tsirkulyar, 1076, 4

Krajci, T. 2005, Inf. Bull. Var. Stars, 5592, 1

Krajci, T. 2006, Inf. Bull. Var. Stars, 5690, 1

Landsman, W., Bohlin, R. C., Neff, S. G., et al. 1998, AJ, 116, 789

Loumos, G. L., \& Deeming, T. J. 1978, Ap\&SS, 56, 285

Lucy, L. B. 1967, ZA 65, 89

Mathieu, R. D., van den Berg, M., Torres, G., et al. 2003, AJ, 125, 246

Millis, R. L. 1972, Inf. Bull. Var. Stars, 649, 1

Milone, A. A. E., \& Latham, D. W. 1992, IAUS, 151, 475

Montgomery, K. A., Marschall, L. A., \& Janes, K. A. 1993, AJ, 106, 181

Nelson, R. H. 2006, Inf. Bull. Var. Stars, 5672, 1

Nelson, R. H. 2007, Inf. Bull. Var. Stars, 5760, 1

Orosz, J. A., \& Hauschildt, P. H. 2000, A\&A, 364, 265

Paczyński, B., Szczygieł, D. M., Pilecki, B., et al. 2006, MNRAS, 368, 1311

Pribulla, T., \& Rucinski, S. M. 2006, AJ, 131, 2986

Pribulla, Th., Rucinski, S., \& Matthews, J. M. 2008, MNRAS, 391, 343

Prša, A., \& Zwitter, T. 2005, ApJ, 628, 426

Qian, S.-B., Liu, L., Soonthornthum, B., Zhu, L.-Y., \& He, J.-J. 2006, AJ, 131, 3028

Rucinski, S. M. 1969, AcA 19, 245

Rucinski, S. M. 1993, PASP, 105, 1433

Shetrone, M. D., \& Sandquist, E. L. 2000, AJ, 120, 1913

Sandage, A., Lubin, L. M., \& VandenBerg, D. A. 2003, PASP, 115, 1187

Sandquist, E. L. 2004, MNRAS, 347, 101

Sandquist, E., \& Shetrone, M. 2001, AAS, 198, 4201

Sandquist, E. L., \& Shetrone, M. D. 2003, AJ, 125, 2173

Sandquist, E. L., Latham, D. W., Shetrone, M. D., et al. 2003, AJ, 125, 810

Sarajedini, A., Brandt, K., Grocholski, A. J., et al. 2004, AJ, 127, 991

Selam, S. O. 2004, A\&A, 416, 1097

Sturch, C. 1973, PASP, 85, 724

Taylor, B. J. 2007, AJ, 133, 370

Tian, B., Deng, L., Han, Z., et al. 2006, A\&A, 455, 247

van den Berg, D. A., \& Stetson, P. B. 2004, PASP, 116, 997

van den Berg, M., Orosz, J., Verbunt, F., et al. 2001, A\&A, 375, 375

van den Berg, M., Stassun, K. G., Verbunt, F., et al. 2002, A\&A, 382, 888

van Hamme, W. 1993, AJ 106, 2096

Whelan, J. A. J., Romanishin, W., Worden, S. P., et al. 1979, MNRAS, 186, 729

Wilson, R. E. 1994, PASP, 106, 921

Wilson, R. E., \& Devinney, E. J. 1971, ApJ, 166, 605

Yakut, K. 2005, Ap\&SS, 296, 273

Yakut, K. 2006, Ph.D. Thesis, Faculty of Science (Leuven, Belgium: KULeuven)

Yakut, K., \& Aerts, C. 2006, in Astrophysics of Variable Stars, ASP Conf. Ser. 349,375

Yakut, K., \& Eggleton, P. P. 2005, ApJ, 629, 1055

Yakut, K., İbanoğlu, C., Kalomeni, B., et al. 2003, A\&A, 401, 1095

Yakut, K., Kalomeni, B., \& Tout, C. A. 2008 [arXiv: 0811. 0455]

Yang, Y.-G., Dai, J.-M., Yin, X.-G., et al. 2007, AJ, 134, 179

Youn, J.-H., Kim, H.-I., Lee, J-W., et al. 2003, J. Astron. Space Sci. 20, 249

Zhang, X. B., Zhang, R. X., \& Deng, L. 2005, AJ, 129, 979

Zima, W. 2008, Comm. Asteroseismol. 155 